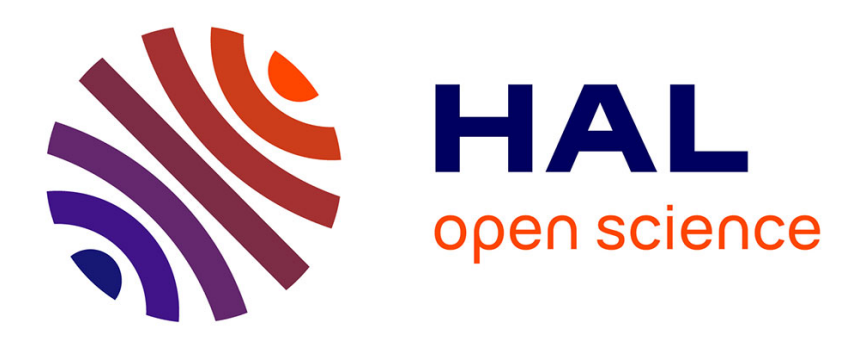

\title{
Inf-structuring functions and self-dual marked flattenings in bi-Heyting algebra
}

\author{
Benjamin Perret
}

\section{To cite this version:}

Benjamin Perret. Inf-structuring functions and self-dual marked flattenings in bi-Heyting algebra. ISMM 2013, May 2013, Uppsala, Sweden. pp.365-376, 10.1007/978-3-642-38294-9_31 . hal00803422

\section{HAL Id: hal-00803422 \\ https://hal.science/hal-00803422}

Submitted on 21 Mar 2013

HAL is a multi-disciplinary open access archive for the deposit and dissemination of scientific research documents, whether they are published or not. The documents may come from teaching and research institutions in France or abroad, or from public or private research centers.
L'archive ouverte pluridisciplinaire $\mathbf{H A L}$, est destinée au dépôt et à la diffusion de documents scientifiques de niveau recherche, publiés ou non, émanant des établissements d'enseignement et de recherche français ou étrangers, des laboratoires publics ou privés. 


\title{
Inf-structuring functions and self-dual marked flattenings in bi-Heyting algebra
}

\author{
Benjamin Perret \\ Université Paris-Est, Laboratoire d'Informatique Gaspard-Monge, Equipe A3SI, ESIEE Paris, \\ 2, boulevard Blaise Pascal \\ Cité Descartes BP 99 \\ 93162 Noisy le Grand CEDEX France \\ perretb@esiee.fr
}

\begin{abstract}
This paper introduces a generalization of self-dual marked flattenings defined in the lattice of mappings. This definition provides a way to associate a self-dual operator to every mapping that decomposes an element into subelements (i.e. gives a cover). Contrary to classical flattenings whose definition relies on the complemented structure of the powerset lattices, our approach uses the pseudo relative complement and supplement of the bi-Heyting algebra and a new notion of inf-structuring functions that provides a very general way to structure the space. We show that using an inf-structuring function based on connections allows to recover the original definition of marked flattenings and we provide, as an example, a simple inf-structuring function whose derived self-dual operator better preserves contrasts and does not introduce new pixel values.
\end{abstract}

Keywords: inf-structuring function, self-dual operator, flattening, Heyting algebra, connection, hyper-connection, image processing, mathematical morphology

\section{Introduction}

Duality is a principle which states that the content of a numerical function remains the same after an inversion. Following this principle, an operator should process an image and its opposite symmetrically, i.e. be self-dual. Although this property is always achieved with linear filters, the problem is harder in mathematical morphology where the operations of infimum and supremum treat differently bright and dark objects.

Several authors have explored various manners to define self-dual morphological operators $[3,4,9,5,20]$. The first approaches are based on the notion of activity of a boolean operator which leads to the activity lattice [15] where given two dual binary operators, both their infimum (morphological centre) and supremum (flattening [7] and levelling $[8,19]$ ) produce self-dual operators. The second approaches rely on the notion of tree of shapes [9,5] which is the tree given by the relation of inclusion between the shapes (level lines) in the image. The definition of shapes and the inclusion relation being invariant to contrast inversion, this naturally leads to self-dual operators. A third approach consists in working with the module of the gradient of the function.

This paper generalizes the first approach and more precisely the marked flattening [18]. Marked flattenings are defined as increasing set operators which are thus 
easily extended to functions by thresholding and stacking. One calls the flattening $\theta_{M}(A)$ of the set $A$ by the set $M$, the union of $\gamma_{M}(A)$, the set of connected components of $A$ that intersect $M$, with the set of all pores (connected components of the background) that do not intersect $M^{c}$, i.e. those which are included in the marker: $\theta_{M}(A)=\gamma_{M}(A) \cup\left(A^{c} \cap \gamma_{M^{c}}^{c}(A)\right)$.

Currently, the definition of the marked flattening is only feasible in sets due to the use of the complementation. We show that the use of the bi-Heyting algebra structure removes this limitation and lets us generalize the flattenings in order to use connections on functions [17]. In order to encompass all possible approaches for connections, in section 3, we define the new notion of inf-structuring function (isf), which is a mapping that associates to any function a set of smaller functions, and we propose two particular isfs. In section 4, we propose two swamping functions based on isfs and we show that it generalizes the classical notion of connected openings and closings. Then, section 5 presents the construction of the self-dual operator combining isf-swampings and Heyting algebra operators. We show that using adapted isfs, either based on connections or on hyper-connections, enables us to recover the original definition of flattenings and to define new self-dual operators that better preserve contrasts and that do not create new pixel values. Finally, we conclude the work in section 7 and we give a few perspectives.

\section{Mathematical preliminaries}

Let $\left(\mathscr{L}, \vee_{\mathscr{L}}, \wedge \mathscr{L}, \mathbf{0}, \mathbf{1}, \leq \mathscr{L}\right)$ be a complete lattice, where $\mathscr{L}$ is the set of elements of the lattice, $\wedge \mathscr{L}$ (resp. $\vee_{\mathscr{L}}$ ) is the infimum (resp. supremum), $\mathbf{0}$ (resp. 1) is the smallest (resp. largest) element and $\leq_{\mathscr{L}}$ is the associated partial order. The lattice $\mathscr{L}$ is infinite $\vee$ distributive (resp. $\wedge$-distributive) if $\forall a \in \mathscr{L}, \forall B \subseteq \mathscr{L}$, we have $a \wedge\left(\bigvee_{b \in B} b\right)=\bigvee_{b \in B}(a \wedge$ b) (resp. $\left.a \vee\left(\bigwedge_{b \in B} b\right)=\bigwedge_{b \in B}(a \vee b)\right)$. It is infinite distributive if it is both infinite $\vee$ and $\wedge$-distributive. Given an element $a \in \mathscr{L}$ we note $M_{a}$ (resp. $M^{a}$ ) the set of upper bounds of $a$ (resp. lower bounds): $M^{a}=\{b \in \mathscr{L} \mid b \leq a\}$ and $M_{a}=\{b \in \mathscr{L} \mid b \geq a\}$

We also define the particular complete infinite distributive lattice of mappings from a non empty set $\mathscr{D}$ to a complete chain $\mathscr{T}:\left(\mathscr{F}, \vee_{\mathscr{F}}, \wedge \mathscr{F}, \perp, \top, \leq \mathscr{F}\right)$, where $\mathscr{F}$ is the set of elements of the lattice, $\wedge_{\mathscr{F}}$ (resp. $\vee_{\mathscr{F}}$ ) is the pointwise infimum (resp. supremum) operator, $\perp$ (resp. $\top$ ) is the smallest (resp. largest) element and $\leq \mathscr{F}$ is the associated partial order. For simplicity of notations, when possible, we omit the indices from the infimum, supremum and ordering symbol. In image processing, we usually define $\mathscr{T}$ as a closed subset of the completed real line $\overline{\mathbb{R}}=\mathbb{R} \cup\{-\infty,+\infty\}$ or $\overline{\mathbb{Z}}=\mathbb{Z} \cup\{-\infty,+\infty\}$.

\subsection{Self-dual operator}

We give here a short presentation of self-duality, a deeper exploration is given in [4].

Definition 1. An operator $\varphi$ of $\mathscr{L}$ is an inversion if it is a decreasing involution (i.e. $\varphi$ is a decreasing, bijective mapping from $\mathscr{L}$ to $\mathscr{L}$, such that $\left.\forall a \in \mathscr{L}, \varphi^{2}(a)=a\right)$.

For simplicity of notation, we write $a^{*}$ for an element $a \in \mathscr{L}$ where we assume that an inversion $\varphi$ exists and that $a^{*}=\varphi(a)$. If the lattice $\mathscr{L}$ is distributive and if an inversion 
$\varphi$ exists for $\mathscr{L}$ then the image of $\mathscr{L}$ by $\varphi$ is the dual lattice of $\mathscr{L}$ obtained by inverting the ordering relation and exchanging the infimum and supremum operators.

If $\mathscr{L}=\overline{\mathbb{R}}($ resp. $\mathscr{L}=\overline{\mathbb{Z}}$ ), an inversion is given by: $\forall a \in \mathscr{L}, \varphi(a)=n-a$ with $n \in \mathbb{R}$ (resp. $n \in \mathbb{Z}$ ). If $\mathscr{L}$ is a closed subset $[n, m]$ of $\mathbb{R}$ or $\mathbb{Z}$, then an inversion is given by: $\forall a \in \mathscr{L}, \varphi(a)=n+m-a$. This extends to the lattice $\mathscr{F}$ using pointwise operations.

Definition 2. Being given an operator $\alpha$ of $\mathscr{L}$, we define the dual operator $\alpha^{*}$ for $\varphi$ by: $\forall a \in \mathscr{L}, \alpha^{*}(a)=\left(\alpha\left(a^{*}\right)\right)^{*}$.

The dual transformation of $a$ is the inverse of the transformation of the inverse of $a$. Duality is a common method to define pairs of operators like openings and closings.

Definition 3. An operator $\alpha$ is self-dual if it is equal to its dual operator: $\alpha=\alpha^{*}$.

A self-dual operator treats an element and its inverse equally. In image processing this can be interpreted as a being covariant to contrast inversion. The convolution and the median filter are two well known examples of self-dual operators.

\subsection{Bi-Heyting algebra}

Heyting algebras are well known in the field of propositional logic, but, to our knowledge, has only been used by Stell et al. in the field of mathematical morphology [22,21].

Definition 4. A complete Heyting algebra is a complete $\vee$-infinite distributive lattice $\mathscr{L}$ with a binary operator pc called relative pseudo-complement such that $\forall a, b \in \mathscr{L}$, $\mathrm{pc}(a, b)$ is the largest element such that $a \wedge \mathrm{pc}(a, b) \leq b$ [23].

The lattice $\mathscr{F}$ is a Heyting algebra with $\forall f, g \in \mathscr{F}, \operatorname{pc}(f, g)=\bigvee\{h \mid f \wedge h \leq g\}$ (Fig. 1). If $\mathscr{L}$ is a complemented $\vee$-infinite distributive lattice, then the mapping $\operatorname{pc}(a, \mathbf{0})$ for $a \in \mathscr{L}$ is indeed the classical complementation.

Definition 5. A complete co-Heyting algebra is a complete $\wedge$-infinite distributive lattice $\mathscr{L}$ with a binary operator $\mathrm{ps}$, called relative pseudo-supplement such that $\forall a, b \in$ $\mathscr{L}, \operatorname{ps}(a, b)$ is the smallest element such that $a \vee \operatorname{ps}(a, b) \geq b[6]$.

The lattice $\mathscr{F}$ is a co-Heyting algebra with $\forall f, g \in \mathscr{F}, \operatorname{ps}(f, g)=\bigwedge\{h \mid f \vee h \geq g\}$.

An algebra that is both a Heyting and a co-Heyting algebra is then referred as a bi-Heyting algebra [12].

ps and pc are dual operators: $\forall a, b \in \mathscr{L}, \operatorname{ps}(a, b)=\left(\mathrm{pc}\left(a^{*}, b^{*}\right)\right)^{*}$ and conversely $\operatorname{pc}(a, b)=\left(\operatorname{ps}\left(a^{*}, b^{*}\right)\right)^{*}$. Fig. 1 shows two examples of applications of $\mathrm{pc}$ and $\mathrm{ps}$ in $\mathscr{F}$. We see that $\mathrm{pc}(f, g)$ is equal to $\top$ where $f$ is smaller than $g$ and to $g$ otherwise, conversely $\operatorname{ps}(f, g)$ is equal to $\perp$ where $f$ is larger than $g$ and to $g$ otherwise.

\section{Inf-structuring functions}

In this section, we present the new concept of isf which is a very general type of mapping that associates to each element of a lattice a set of sub-elements (Fig. 2) 

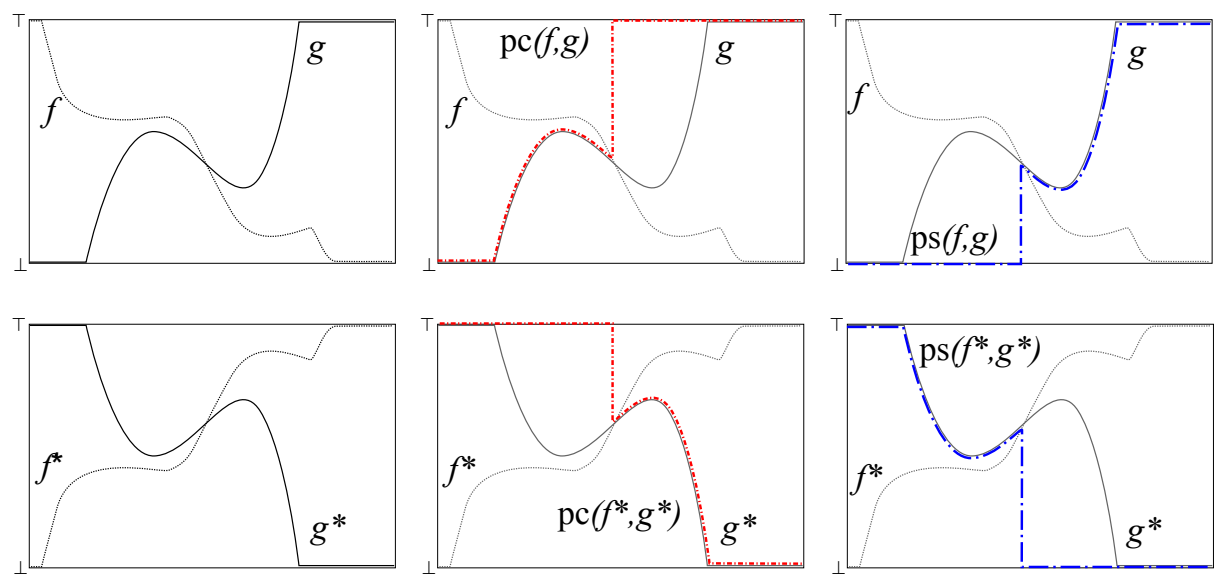

Fig. 1. Illustration of the relative pseudo-complement and the relative pseudo-supplement in $\mathscr{F}$. The first line shows the application of the relative pseudo-complement $\mathrm{pc}$ and the relative pseudosupplement ps on two functions $f$ and $g$. The second line shows the duality between pc and ps.

Definition 6. We say that $\mathrm{s}: \mathscr{F} \mapsto \mathscr{P}(\mathscr{F})$ is an inf-structuring function (isf) of $\mathscr{F}$ if:

1. $\forall f \in \mathscr{F}, \mathrm{s}(f) \subseteq M^{f}$ (i.e. $\forall g \in \mathrm{s}(f), g \leq f$ ): all sub-elements are smaller than $f$.

2. $\forall f \in \mathscr{F}, \bigvee \mathrm{s}(f)=f$ : the supremum of the sub-elements of $f$ is equal to $f$

One can note that an important difference with (hyper-)connections is that an isf can decompose an element into comparable elements.

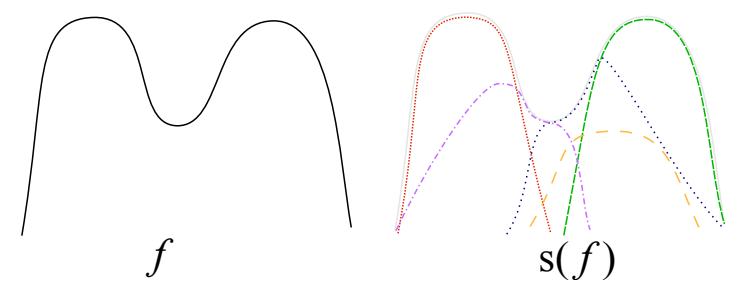

Fig. 2. Example of decomposition of a function $f$ into a set of five lower functions $s(f)$. The assumptions made on the content of $\mathrm{s}(f)$ are very weak.

A simple way of constructing isfs is to use the tool-box from the connection theory. We present here two isfs, the first one based on the set connections [16] and the second one the hyper-connections [17].

\subsection{Set connections for isf}

Set connections are a convenient way to describe how elements of a set are grouped in so called connected components [16]. Being given a connection $\mathscr{C}$ on the powerset lattice 
$\mathscr{P}(E)$, a subset $A$ of $E$ and a point $x$ in $E$, the connected component of $A$ containing $x$ (for $\mathscr{C}$ ), noted $\gamma_{x}(A)$, is the largest element of $\mathscr{C}$ containing $x$ and included in $A$. We can now define the isf based on the connection $\mathscr{C}$ and denoted by s $\mathscr{C}$ as:

$$
\forall f \in \mathscr{F}, \mathrm{s}_{\mathscr{C}}(f)=\left\{\bigvee_{v \leq t} \operatorname{cyl}\left(\gamma_{x}\left(\bar{f}^{v}\right), v\right) \mid t \in \mathscr{T}, x \in \mathscr{D}\right\}
$$

where $\operatorname{cyl}(X, v)$ represents the cylinder of base $X$ and level $v$ : for all $x \in \mathscr{D}, \operatorname{cyl}(X, v)(x)$ equals $v$ if $x \in X$ and $\mathbf{0}$ otherwise. And, $\vec{f}^{t}$ is the threshold of the function $f \in \mathscr{F}$ at level $t \in \mathscr{T}$, i.e. the set of points where the value of $f$ is larger than $t: \vec{f}^{t}=\{x \in \mathscr{D} \mid f(x) \geq t\}$.

This construction, depicted in Fig. 3, is indeed closely linked (see Prop. 1) to the definition of anti-extensive connected operators in gray-level images [14].

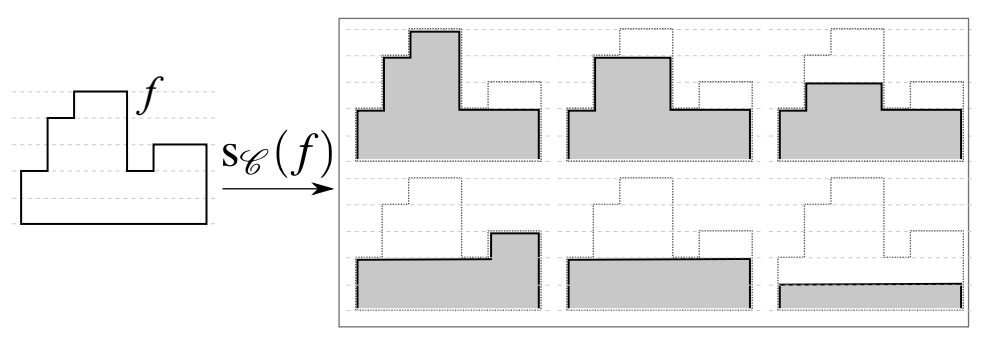

Fig. 3. Set connection based isf: the function on the left is decomposed into the 6 functions in the right box. We assume here that the value domain is discrete (dashed horizontal lines).

\subsection{Ultimate flat zone isf}

We define here the ultimate flat zone isf noted $\mathrm{s}_{u f z}$. Each element of the isf of $f$ corresponds to the infimum between a maximal flat function of $f$ (i.e. a flat function with connected support such that there do not exist another flat function strictly larger than it and lower than $f$ ) and a flat zone of $f$ (Fig. 4). This construction can be formalized with hyper-connections [17] as an iterative decomposition into z-zones [1,11] using the h-connection of flat functions [10].

\section{Isf swampings}

The definition of the generalized flattening is done in two steps. We first give a generalized definition of a swamping (marked reconstruction) based on the notion of isf. Then, in the next section, we define the generalized flattening using the swamping function and the bi-Heyting algebra.

An isf provides a first way to structure the space by considering a notion of local minima conditionally to the decomposition. We define an elementary swamping function, i.e. a marked reconstruction, $\beta: \mathscr{F} \times \mathscr{F} \mapsto \mathscr{F}$ by:

$$
\beta(f, m)=\bigvee \min \left(M_{m} \cap \mathrm{s}(f)\right)
$$




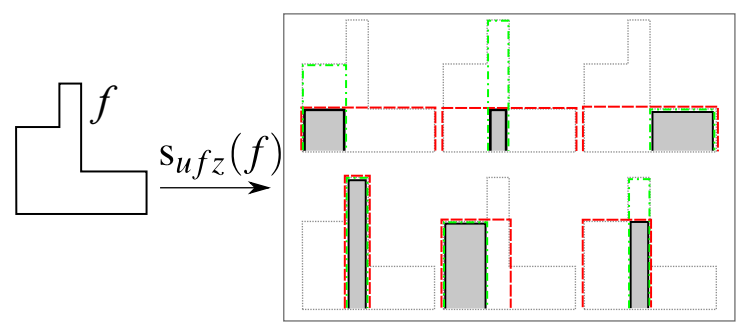

Fig. 4. Ultimate flat zone isf: the function on the left is decomposed into 6 functions showed in the right box. Each element of the isf is given by the infimum between a maximal flat function (red dashed line) and a flat zone (green dot-dash line).

where $f$ is the processed element, $m$ is the marker and $\min (X)$ is the set of minimal elements of the set $X(\min (X)=\{x \in X \mid \forall y \in X, y \leq x \Rightarrow y=x\})$. The use of the min in this formula insures that for each element $g$ of s $(f)$ there exists a marker $m$ such that $g=\beta(f, m)$.

Then, we define the isf-swamping $\alpha: \mathscr{F} \times \mathscr{F} \mapsto \mathscr{F}$ as an extension of $\beta$ :

$$
\forall f, m \in \mathscr{L}, \alpha(f, m)=\bigvee_{n \leq m} \beta(f, n)
$$

The operators $\alpha$ and $\beta$ and their differences are illustrated in Fig. 5. Observe that the purple sub-element (dot-dash line) is included in $\alpha(f, m)$ but not in $\beta(f, m)$ as it only intersects $m$. Also, in both cases, the blue sub-element (largely spaced dotted line) that also intersects $m$ is not included because there does not exist a $n \in M^{m}$ such that $n$ is smaller than this sub-element but larger than the orange one (spaced dashed line).
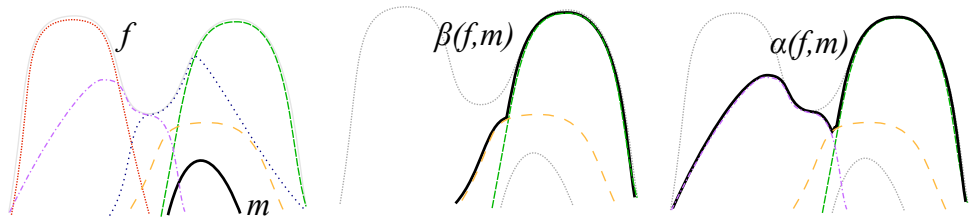

Fig. 5. Example of application of the $\beta$ and $\alpha$ operators on the function $f$ decomposed in $\mathrm{s}(f)$ and marked by $m$. Whereas $\beta$ selects the lowest sub-elements that are greater than the marker, $\alpha$ selects the lowest sub-elements whose support intersects the support of the marker.

The behaviour of $\alpha$ and its dual operator are illustrated in Fig. 6. $\alpha$ is similar to a rasing of $f$ marked by $m$ as it removes all sub-elements of $a$ that are completely outside the marker. On the other side, the dual operator $\alpha^{*}$ acts as a flooding of $f$ marked by $m$, adding all sub-elements of the inverse that are completely included in the marker.

$\alpha$ is not increasing with respect to $f$ (Fig. 7) but it is trivially increasing with respect to $m$ as more and more sub-elements are included in the result when $m$ increases. The fact that $\alpha$ is not increasing with respect to its first argument is not a real drawback as 


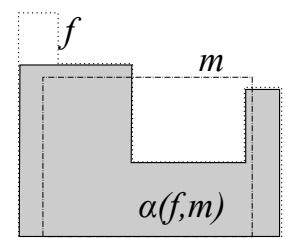

(a)

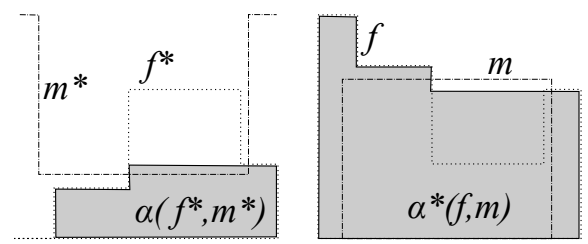

(b)

Fig. 6. Illustration of the $\alpha$ operator and its dual operator with the ultimate flat zone isf (Fig. 4). Fig. (a) shows a function $f$ (dotted line) a marker $m$ (dot-dash line) and the result $\alpha(f, m)$ (in grey). The first image of Fig. (b) shows the result of $\alpha\left(f^{*}, m^{*}\right)$. Finally, the last image shows the result of the dual operator $\alpha^{*}(f, m)$.

this property is indeed really important only for binary operators in order to naturally extend them to the grayscale case by stacking. It is clearly anti-extensive with respect to $f$ but not to $m$. It is generally not idempotent with respect to $f$.

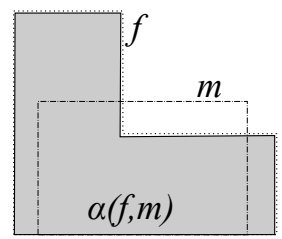

(a)

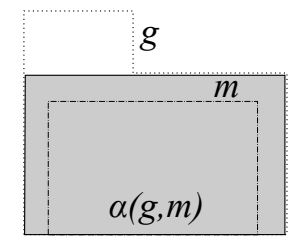

(b)

Fig. 7. Example showing that $\alpha$ is generally not increasing with the ultimate flat zone isf (Fig. 4). Fig. (a) shows the result of $\alpha(f, m)$, whereas we have the result of $\alpha(g, m)$ on Fig. (b). We have $f \leq g$ but $\alpha(f, m)$ and $\alpha(g, m)$ are not comparable.

Proposition 1. When the isf is based on connection ( $\mathrm{s}_{\mathscr{C}}:$ Eq. 1), then $\alpha$ reduces to a classical marked connected opening (and closing for $\alpha^{*}$ ).

\section{Generalized flattenings}

Based on $\alpha$, we can construct a new self-dual operator $\Theta: \mathscr{L} \times \mathscr{L} \mapsto \mathscr{L}$ following the method used for the definition of binary flattenings [7,18]. Nevertheless, binary flattenings rely on the complemented structure of the binary lattice and we will show that this construction can be extended using the notion of relative pseudo-complement and -supplement. Formally, $\Theta$ is defined by:

$$
\forall f, m \in \mathscr{L}, \Theta(f, m)=\alpha(f, m) \vee \operatorname{ps}\left(f, \alpha^{*}(f, m)\right) .
$$

This equation is illustrated in Fig. 8. Its first part: $\alpha(f, m)$ takes the supremum of the smallest sub-elements of $f$ that intersect $m$. Conversely, $\alpha^{*}(f, m)$ takes the inverse of the supremum of the smallest sub-elements of $f^{*}$ that intersect $m^{*}$ which 


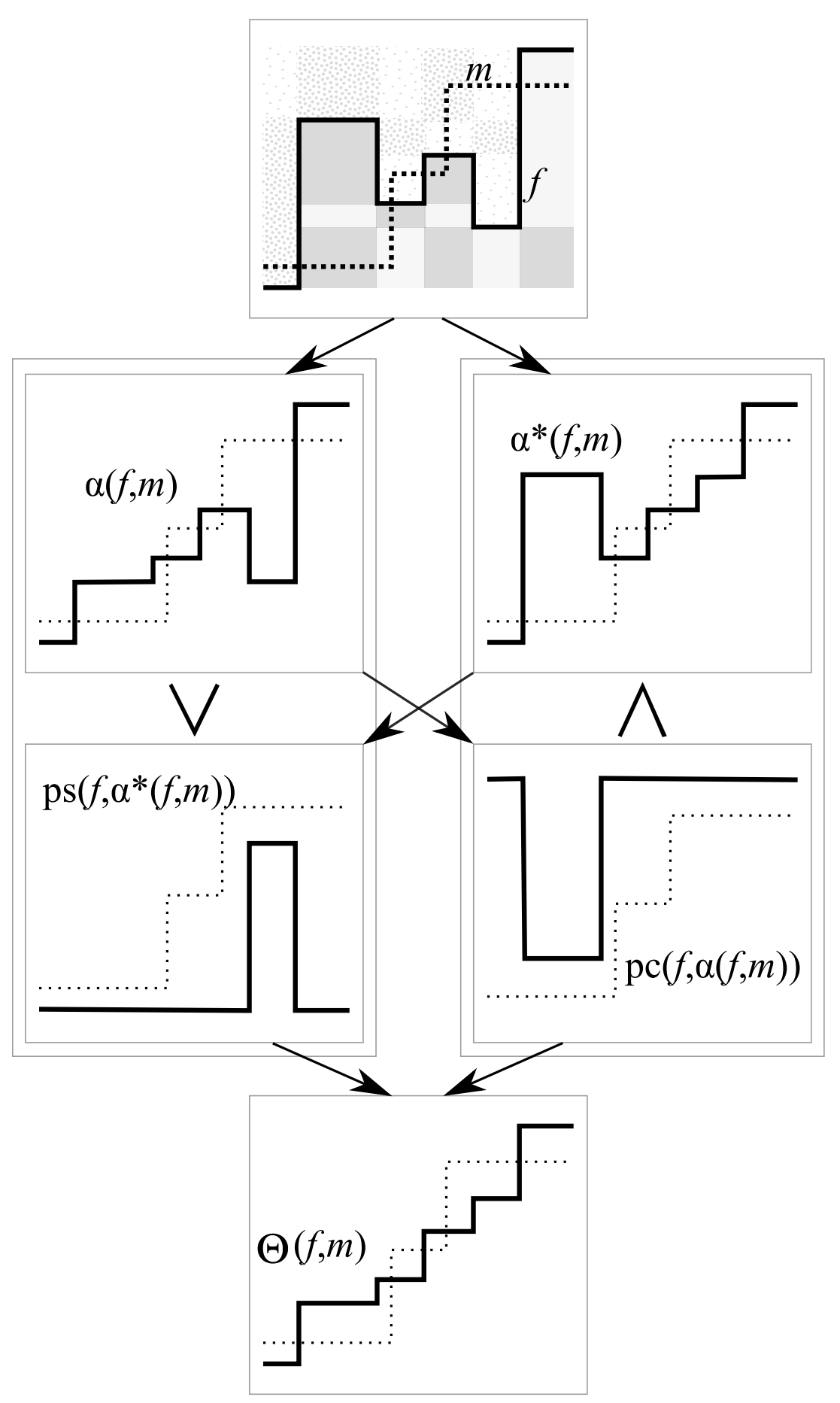

Fig. 8. Example of application of the operator $\Theta(f, m)=\alpha(f, m) \vee \operatorname{ps}\left(f, \alpha^{*}(f, m)\right)$ (Eq. 4) and its equivalent definition $\Theta(f, m)=\alpha^{*}(f, m) \wedge \mathrm{pc}(f, \alpha(f, m))$ (Prop. 2) using an ultimate flat zone isf (Fig. 4). 
can be interpreted as adding to $f$ the "parts" of $f^{*}$ that are completely included in $m$. Then $\operatorname{ps}\left(f, \alpha^{*}(f, m)\right)$ filters $\alpha^{*}(f, m)$ in order to only keep those added parts. Finally, $\Theta(f, m)$ is the supremum between the filtered version $\alpha(f, m)$ and the added parts from $\alpha^{*}(f, m)$. Intuitively the final result is composed of the parts of $f$ that intersect $m$ and the parts of $f^{*}$ that are below $m$.

Proposition 2. For all $f, m \in \mathscr{L}$, we have $\Theta(f, m)=\alpha^{*}(f, m) \wedge \mathrm{pc}(f, \alpha(f, m))$

This equation is illustrated in Fig. 8. Here, $\alpha^{*}(f, m)$ adds to $f$ the "parts" of $f^{*}$ that are completely included in $m$. On the other side, $\mathrm{pc}(f, \alpha(f, m))$ determines which parts of $f$ are not selected by $\alpha(f, m)$, i.e. those that do not intersect $m$. Finally we remove from $\alpha^{*}(f, m)$ the parts extracted by $\operatorname{pc}(f, \alpha(f, m))$.

Proposition 3. $\Theta$ is a self-dual operator: $\Theta(f, m)=\Theta\left(f^{*}, m^{*}\right)^{*}$

This is an immediate consequence of Prop. 2 and the duality between pc and ps.

Proposition 4. When the isf is based on connection ( $\mathrm{s} \mathscr{C}:$ Eq. 1), then $\Theta$ reduces to the classical connected marked flattening operator as defined in [18].

This is a direct consequence of Prop. 1.

\subsection{Discussion}

It is also possible to interpret $\Theta$ as the supremum of activity between $\alpha$ and $\alpha^{*}$ which corresponds to the fact that binary marked flattenings can themselves be defined as the supremum of activity of two operators. Nevertheless, this previous definition was only feasible in complemented lattices while it is here directly expressed in the lattice of mappings thanks to the bi-Heyting algebra structure. One can note that Serra touched upon this construction with the Lemma 8.2 of [15] where he noticed that the use of an infinite-distributive lattice was usefull in this frame.

One can note that $\Theta$ is not a generalization of marked levellings of [19] because the later rely on two particular relations. In [19] a component of the foreground is selected if it touches the marker and at the opposite a component of the background is selected if it is strictly included in the marker. While in our definition, a part of the function is selected if it intersects the marker and at the opposite a part of the inverse of the function is selected it is under the marker. While the relations used in [19] ensure that the resulting operator is adjacency stable we do not have this property and $\Theta$ is thus not a levelling [2]. Nevertheless, if we use two different markers in the definition of $\Theta$, then, we can recover the classical marked leveling by taking the dilation of $m$ as the marker of $\alpha$ and the erosion of $m$ as the marker of $\alpha^{*}$.

One can also note that the self-duality of the complete application of the operator (computation of $m$ and application of $\Theta$ ) is indeed only verified if the marker is computed with a self-dual operator. The question of the real properties of morphological operators based on context information (marker for flattenings and levelings, set of structural elements for spatially variant morphology) has been discussed by several authors $[13,19]$ and more extensively in [2]. 


\section{Applications}

We propose applications to exhibit some differences between the self-dual operator $\Theta$ obtained with the ultimate flat zone isf and the classical flattenings or levelings.

The first line of Fig. 9 illustrates the fact that $\Theta$ cannot introduce new pixel values with the ultimate flat zone isf. The original image is a simple chess board while the marker is the result of a convolution of the chess board with a Gaussian kernel. With the classical leveling new pixel values coming from the marker are introduced in the result while in our new definition, the result is equal to the original image. The second line of Fig. 9 relies this time on a noisy version of the chess board image. Similarly to the classical leveling, our operator reduces drastically the noise level, does not move frontiers and moreover, it also better preserves the contrast. A profile view of the same example is given Fig. 10.
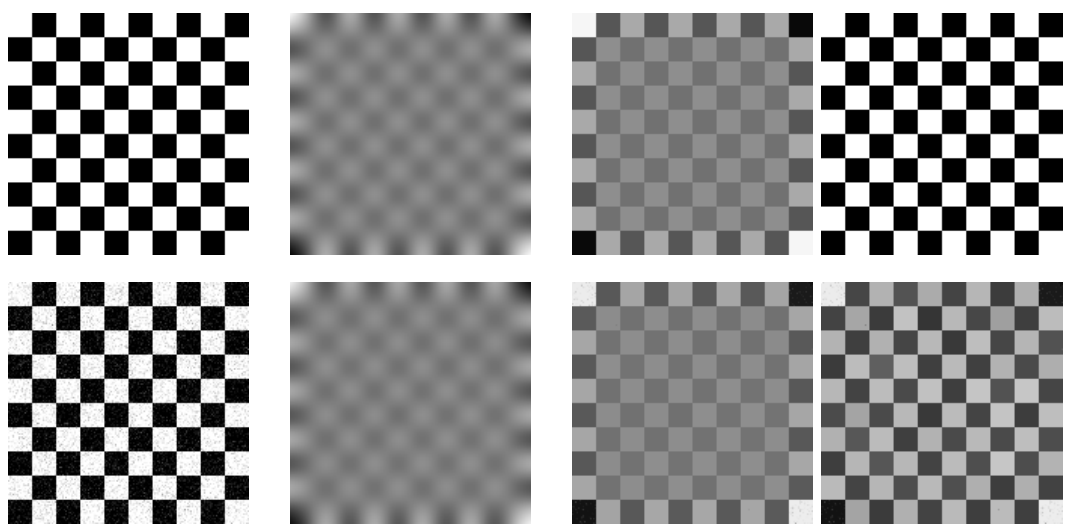

Original $a$

Marker $m$ (Convolution)

Leveling

$\Theta(a, m)$

Fig. 9. Comparison of the generalized flattening $\Theta$ with the classical levelling. First line: the original image does not contain any noise, we see here that the use of the ultimate flat zone isf prevents the introduction of new grey levels in the result. Second line: here the original image is noisy and we show that $\Theta$ better preserves the contrast as it tends to reconstruct the maxima.

Finally, Fig. 11 shows a real life application on a picture of Uppsala. One can observe that our operator performs a simplification of the image that is similar to the one provided by the connected leveling while offering a better preservation of the contrast.

\section{Conclusion}

In this article we have proposed a generalization of the self-dual marked flattenings that provides a way to associate a self-dual operator to every mapping that decomposes an element into sub-elements. It is defined directly in the lattice of mappings thanks to its bi-Heyting algebra structure and it relies on the new notion of inf-structuring functions. We derive two new swampings from the notion of isf and we show that they 


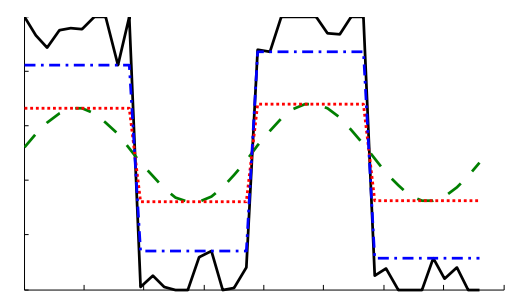

Fig. 10. Illustration of a profile through the images of the second line of Fig. 9. The black solid curve is the original image, the green dashed line is the marker, the red dotted line is the classical leveling and the blue dotted-dashed line is the result of $\Theta$ (built upon the ultimate flat zone isf). We see that $\Theta$ tends to reconstruct the maxima and thus better preserves the contrast.

generalize the connected openings. Then, we define a new self-dual operator based on those swampings that generalizes the marked flattenings. Finally, we show that using a naive isf allows to better preserve the contrast, which suggests that even better self-dual operators can be obtained through the definition of more sophisticated isfs.

In future work, we plan to explore two directions: first the possibility to develop the notion of activity based on the bi-Heyting algebra structure and, second, the deep exploration of the notion of inf-structuring functions and more precisely its various links with connections, hyper-connections and the associated (h-)connected operators.

Acknowledgements I would like to thank Prof. Jean Serra for his valuable comments and corrections on the initial version of this paper.

\section{References}

1. U. Braga-Neto and J. Goutsias. A theoretical tour of connectivity in image processing and analysis. JMIV, 19(1):5-31, 2003.

2. J. Crespo. Adjacency stable connected operators and set levelings. IVC, 28(10):1483-1490, 2010.

3. H.J.A.M. Heijmans. Self-dual morphological operators and filters. JMIV, 6:15-36, 1996.

4. H.J.A.M. Heijmans and Renato Keshet. Inf-semilattice approach to self-dual morphology. JMIV, 17(1):55-80, 2002.

5. R. Keshet. Shape-tree semilattice. JMIV, 22(2-3):309-331, 2005.

6. F.W. Lawvere. Intrinsic co-heyting boundaries and the leiniz rule in certain toposes. In Category Theory, volume 1488 of $L N M$, pages 279-281. Springer-Verlag, 1991.

7. F. Meyer. From connected operators to levelings. In Mathematical Morphology and its applications to image and signal processing, pages 191-198. Kluwer, 1998.

8. F. Meyer. The levelings. In Mathematical Morphology and its applications to image and signal processing, pages 199-206. Kluwer, 1998.

9. P. Monasse and F. Guichard. Fast computation of a contrast-invariant image representation. IEEE TIP, 9(5):860-872, 2000.

10. B. Perret, S. Lefèvre, and C. Collet. Toward a new axiomatic for hyper-connections. In ISMM, volume 6671 of LNCS, pages 85-95. Springer, 2011.

11. B. Perret, S. Lefèvre, C. Collet, and É. Slezak. Hyperconnections and hierarchical representations for grayscale and multiband image processing. IEEE TIP, 21(1):14-27, jan 2012. 

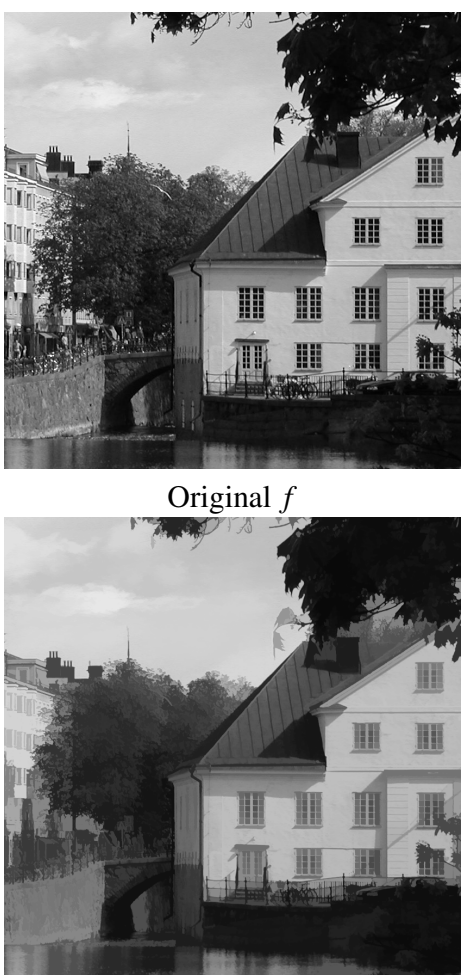

Marked leveling

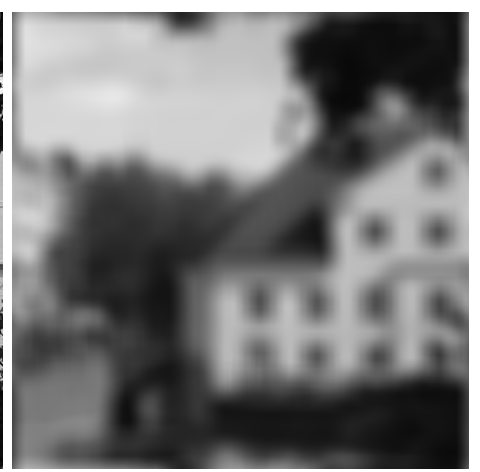

Marker $m$ (Convolution)

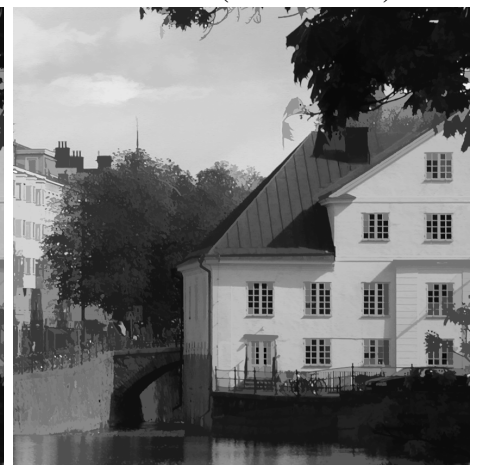

$\Theta(f, m)$

Fig. 11. Comparison of $\Theta$ (built upon the ultimate flat zone isf) with to the connected leveling.

12. G.E. Reyes and H. Zolfaghari. Bi-heyting algebras, toposes and modalities. Journal of Philosophical Logic, 25:25-43, 1996.

13. J.B.T.M. Roerdink. Adaptivity and group invariance in mathematical morphology. In IEEE ICIP'09, pages 2253-2256, 2009.

14. P. Salembier, A. Oliveras, and L. Garrido. Anti-extensive connected operators for image and sequence processing. IEEE TIP, 7(4):555-570, 1998.

15. J. Serra. The centre and self-dual filtering. In Image Analysis and Mathematical Morphology. II: Theoretical Advances, pages 159-180. Academic Press, 1988.

16. J. Serra. Mathematical morphology for boolean lattices. In Image Analysis and Mathematical Morphology. II: Theoretical Advances, pages 37-58. Academic Press, 1988.

17. J. Serra. Connectivity on complete lattices. JMIV, 9(3):231-251, 1998.

18. J. Serra. Connections for sets and functions. Fundam. Inform., 41:147-186, 2000.

19. J. Serra, C. Vachier, and F. Meyer. Levelings. In Mathematical Morphology, pages 199-228. ISTE and John Wiley \& Sons, 2010.

20. P. Soille. Beyond self-duality in morphological image analysis. IVC, 23:249-257, 2005.

21. J.G. Stell. Relations in mathematical morphology with applications to graphs and rough sets. In COSIT, volume 4736 of LNCS, pages 438-454. Springer, 2007.

22. J.G. Stell and M.F. Worboys. The algebraic structure of sets of regions. In COSIT, volume 1329 of LNCS, pages 163-174. Springer, 1997.

23. S. Vickers. Topology via Logic. Cambridge University Press, 1989. 